\title{
Embedding theory as new geometrical mimetic gravity
}

\author{
S. A. Paston ${ }^{\mathrm{a}} \mathbb{0}$, A. A. Sheykin \\ Saint Petersburg State University, Saint Petersburg, Russia
}

Received: 1 August 2018 / Accepted: 21 November 2018 / Published online: 1 December 2018

(C) The Author(s) 2018

\begin{abstract}
It is well known that the recently proposed model of mimetic gravity can be presented as general relativity with an additional mimetic matter. We discuss a possibility to analogously reformulate the embedding theory, which is the geometrical description of gravity proposed by Regge and Teitelboim, treating it also as general relativity with some additional matter. We propose a form of action which allows one to describe this matter in terms of conserved currents. This action turns out to be a generalization of the perfect fluid action, which can be useful in the analysis of the properties of the additional matter. On the other side, the action contains a trace of the root of the matrix product, which is similar to the constructions appearing in bimetric theories of gravity. The action is completely equivalent to the original embedding theory, so it is not just some artificial model, but it has a clear geometric meaning. We discuss the possible equivalent forms of the theory and ways of studying the equations of motion that appear.
\end{abstract}

\section{Introduction}

The idea of mimetic gravity was proposed 5 years ago in Ref. [1] and became quite popular in recent years. In this framework the conformal degree of freedom of gravity is isolated by introducing a parametrization of the physical metric in the following form:

$g_{\mu \nu}=\tilde{g}_{\mu \nu} \tilde{g}^{\gamma \delta}\left(\partial_{\gamma} \lambda\right)\left(\partial_{\delta} \lambda\right)$.

The authors use the familiar form of the General Relativity (GR) action, which consists of the Einstein-Hilbert gravitational action

$S^{\mathrm{EH}}\left[g_{\mu \nu}\right]=-\frac{1}{2 \varkappa} \int d^{4} x \sqrt{-g} R\left(g_{\mu \nu}\right)$

with respect to physical metric $g_{\mu \nu}$, and the matter action $S_{\mathrm{m}}[g]$. However, independent variables in this action are the

a e-mail: s.paston@spbu.ru auxiliary metric $\tilde{g}_{\mu \nu}$ and the scalar field $\lambda$ rather than $g_{\mu \nu}$. The field equations are

$G^{\mu \nu}=\varkappa\left(T^{\mu \nu}+n u^{\mu} u^{\nu}\right), D_{\mu}\left(n u^{\mu}\right)=0, g^{\mu v} u_{\mu} u_{v}=1$

( $T^{\mu \nu}$ is the energy momentum tensor (EMT) of the matter, $D_{\mu}$ is the covariant derivative, the signature is +--- ) where the following notation is used:

$n \equiv g_{\mu \nu}\left(\frac{1}{\varkappa} G^{\mu \nu}-T^{\mu \nu}\right), \quad u_{\mu} \equiv \partial_{\mu} \lambda$.

Since the field equations of mimetic gravity have the form of the Einstein equations with an additional contribution to the EMT corresponding to mimetic matter, one can say that “" dark matter' without dark matter, which is imitated by extra scalar degree of freedom of the gravitational field" [1] arises in the theory.

As can be seen from the field equations, the appearing mimetic matter has the form of a pressure-free perfect fluid with potential motion. It allows one to write an equivalent formulation of mimetic gravity, where the physical metric is considered to be independent, and an additional action term corresponding to such a perfect fluid is present. For the first time this was done in Ref. [2], where the full action is chosen to be

$S=S^{\mathrm{EH}}+S_{\mathrm{m}}+S^{\mathrm{add}}$

where

$S^{\text {add }}=-\frac{1}{2} \int d^{4} x \sqrt{-g}\left(1-g^{\mu \nu}\left(\partial_{\mu} \lambda\right)\left(\partial_{\nu} \lambda\right)\right) n$.

In such a framework the mimetic matter is described by two scalar fields: $n$ can be treated as the number density of mimetic matter particles and $\lambda$ defines their velocity $u_{\mu}=\partial_{\mu} \lambda$. There are other ways of choosing the additional term in the action for a potentially moving pressure-free perfect fluid; see [3]. 
There are also many other ways to write an action for mimetic gravity; see, e.g., [4,5].

The framework of mimetic gravity can be modified to explain certain phenomena of modern cosmology. In particular, the introduction of a potential for the scalar field $\lambda$ [6] leads to the appearance of a pressure of the mimetic matter (note that the structure of the action used appears to be a particular case of the general expression which was already considered in the earlier work [7]), whereas the addition of a higher-order derivative term transforms the mimetic matter into an imperfect fluid $[6,8,9]$. It is also possible to write an action of the mimetic matter in a form with which it turns out to be pressure-free perfect fluid moving arbitrarily (i.e. not potentially anymore) [3]. In the latter case the description of the mimetic matter contains two more fields in addition to $n$ and $\lambda$. Moreover, one can return to the original formulation of mimetic gravity with the auxiliary metric $\tilde{g}_{\mu \nu}$ and three scalar fields that are present in the expression for physical metric $g_{\mu \nu}$ analogous to (1); see for details [3]. For the current status of the mimetic gravity framework see the review [10] and the references therein.

The transformations (1) on which the mimetic gravity is based are a special case of the more general "disformations" [11], which are invertible in the general case, i.e. the auxiliary metric $\tilde{g}_{\mu \nu}$ can be expressed through the physical metric $g_{\mu \nu}$ and the scalar field $\lambda$. It leads to the fact that in the general case of such a change of variables the theory turns out to be equivalent to GR [12]. On the contrary, the special transformations (1) are non-invertible because the conformal mode of $\tilde{g}_{\mu \nu}$ does not contribute to $g_{\mu \nu}$, i.e. the conformal invariance appears [4]. Hence the mimetic gravity is an example of a theory modification appearing as a result of the change of variables that contains differentiation, while the number of variables remains unchanged as 10 components of $g_{\mu v}$ is replaced by 9 components $\tilde{g}_{\mu \nu}$ (without the conformal mode) and the scalar $\lambda$.

This modification is caused by the change of the class of variations of independent field variables, which alters the set of possible solutions of the theory according to the property of the variational principle. In the case of the change of variables that do not contain a differentiation, the change of the class of variations does not occur (of course, if the change of variables does not affect the number of degrees of freedom), so the theory remains unchanged. An example of such a case is a transition from the metric formulation of GR to the tetrad one. Also, if the change of variables contains only spatial derivatives, then the theory usually is not affected due to the common assumption that at spatial infinity the fields decrease sufficiently fast. Therefore the presence of time derivatives in the change of variables leads to a significant modification of the theory. Since the class of variations becomes smaller after such a change (the variation of an independent variable is always assumed to vanish at the initial and final moments of time, so this requirement poses an additional restriction on the original variable), the set of solutions of the modified theory turns out to be larger, including all the solutions of the original theory. It is precisely so in the case of mimetic gravity: the solutions of (3) at $n=0$ (which corresponds to the absence of mimetic matter) are the ones of GR. A simple example of a change of variables with differentiation in 0dimensional (i.e. mechanical) theory can be found in [13].

Another example of a change of variables in GR that contains differentiation has been known for a long time: it is the Regge-Teitelboim gravity [14] also known as the embedding theory. In contrast with mimetic gravity (1) the underlying change of variables in the embedding theory approach has a deep geometric meaning. In this string-inspired approach the assumption is made that our spacetime is not only an abstract pseudo-Riemannian space, but rather a surface in a flat $N$-dimendional ambient space (bulk). Consequently, the induced metric appears on the surface

$g_{\mu \nu}=\left(\partial_{\mu} y^{a}\right)\left(\partial_{\nu} y^{b}\right) \eta_{a b}$,

where $\eta_{a b}$ is a flat ambient space metric, $a, b=0, \ldots, N-1$. This relation defines the change of variables and plays a role analogous to (1), expressing the physical metric through the embedding function $y^{a}(x)$, which turns out to be analogous to auxiliary quantities $\tilde{g}_{\mu \nu}$ and $\lambda$ of mimetic gravity. If one does not wish to lower the number of degrees of freedom, one must take $N \geq 10$ (since 4D metric has 10 independent components). The Friedmann-Janet-Cartan theorem [15] gives the same restriction: it states that an arbitrary $(3+1) \mathrm{D}$ pseudoRiemannian spacetime can be locally isometrically embedded into a flat Minkowski spacetime of no less than 10 dimensions, of which at least one is timelike and at least three are spacelike. Usually $(9+1) D$ Minkowski space is taken as the ambient space of the embedding theory.

For the action of the embedding theory one has the same expression, $S\left[g_{\mu \nu}\right]=S^{\mathrm{EH}}\left[g_{\mu \nu}\right]+S_{\mathrm{m}}\left[g_{\mu \nu}\right]$, as in mimetic gravity, but the metric in it is given by (7) instead of (1). Variation w.r.t. independent variable $y^{a}$ leads to the field equations in the Regge-Teitelboim form,

$D_{\mu}\left(\left(G^{\mu \nu}-\varkappa T^{\mu \nu}\right) \partial_{\nu} y^{a}\right)=0$,

which, besides GR solutions, also admit other solutions, known as extra solutions. After the original paper [14] the ideas of embedding theory were discussed in detail in [16], and later were used in numerous papers devoted to the various aspects of gravity, including quantization; see, e.g., [17-26]. A detailed bibliography of embedding theory and closely related topics can be found in [27].

As in mimetic gravity, the field equation (8) of embedding theory can be written $[13,28]$ in the form of Einstein ones with an additional contribution $\tau^{\mu \nu}$ to the EMT which 
corresponds to certain fictional embedding matter:

$G^{\mu \nu}=\varkappa\left(T^{\mu \nu}+\tau^{\mu \nu}\right)$,

supplemented by the condition (7) and the equation

$D_{\mu}\left(\tau^{\mu \nu} \partial_{\nu} y^{a}\right)=0$.

Equations (7) and (10) can be interpreted as the equations of motion of embedding matter described by the quantities $\tau^{\mu \nu}$ and $y^{a}$. It can be shown (see, for example, [22]) that the standard condition of covariant conservation of the embedding matter EMT, $D_{\mu} \tau^{\mu \nu}=0$, follows from these equations.

Several questions can be raised: how should one write an action of the embedding theory in the form of GR with additional embedding matter, what is this matter like, and what are its laws of motion from the physical point of view? The most straightforward way to answer the first question was proposed in [3], but the choice of independent variables made there does not allow one to say anything related to the second question. In the present work we will consider some alternative ways of choosing the action of embedding theory, as well as of choices of independent variables for the description of embedding matter which is more convenient in a discussion of its physical meaning.

\section{Forms of action for embedding matter}

One can write such an action of the embedding matter $S^{\text {add }}$, for the full action

$S=S^{\mathrm{EH}}+S_{\mathrm{m}}+S^{\text {add }}$,

leading to the field equations of the embedding theory (7), (9), (10), in the most simple manner by satisfying the condition (7) through a Lagrange multiplier [3]:

$S_{1}^{\text {add }}=\frac{1}{2} \int d^{4} x \sqrt{-g}\left(\left(\partial_{\mu} y^{a}\right)\left(\partial_{\nu} y_{a}\right)-g_{\mu \nu}\right) \tau^{\mu \nu}$.

It is easy to check that the variation w.r.t. $\tau^{\mu \nu}$ leads to Eq. (7), whereas w.r.t. $y^{a}$ it leads to (10). ${ }^{1}$ However, the physical meaning of the variables $\tau^{\mu \nu}$ and $y^{a}$ which describe the embedding matter in such an approach remains unclear.

Let us consider an alternative form of the contribution of the embedding matter to the action, namely

$S_{2}^{\mathrm{add}}=\int d^{4} x \sqrt{-g}\left(j_{a}^{\mu} \partial_{\mu} y^{a}-\operatorname{tr} \sqrt{g_{\mu \nu} j_{a}^{v} j^{\alpha a}}\right)$,

where the operation $\sqrt{ }$ means taking the root of the matrix with indices $\mu$ and $\alpha$ and $\operatorname{tr}$ means subsequently taking the trace. The independent variables describing the embedding

\footnotetext{
1 Note that an analogous way of addition of $S_{1}^{\text {add }}$ to the Nambu-Goto action leads to the Polyakov string action; see Eqs. (31)-(35).
}

matter in this approach (which we will call embedding gravity) are the quantities $j_{a}^{\mu}$ and $y^{a}$.

Let us write the equations of motion that appear. The variation w.r.t. $y^{a}$ immediately leads to the equation

$D_{\mu} j_{a}^{\mu}=0$.

Now let us find the variation of (13) w.r.t. $j_{a}^{\mu}$. To do this, define

$A_{\mu}^{\alpha}=g_{\mu \nu} j_{a}^{v} j^{\alpha a}-\delta_{\mu}^{\alpha}$.

Also denote the result of taking the root in (13) as $\beta_{\mu}{ }^{\alpha}$, so in the index-free notation

$\beta=\sqrt{I+A}$,

where $I$ is an identity matrix. Then for the variation we have

$$
\begin{aligned}
\beta^{2}=I+A \Rightarrow & (\delta \beta) \beta+\beta \delta \beta=\delta A \\
& \Rightarrow \quad \beta^{-1}(\delta \beta) \beta+\delta \beta=\beta^{-1} \delta A \\
& \Rightarrow \quad \delta \operatorname{tr} \beta=\frac{1}{2} \operatorname{tr}\left(\beta^{-1} \delta A\right) .
\end{aligned}
$$

Note that accordingly to the chosen notations the quantity $A^{\nu \alpha}=g^{\nu \mu} A_{\mu}^{\alpha}=j_{a}^{v} j^{\alpha a}-g^{\nu \alpha}$ turns out to be symmetric. If the matrix root is treated as a Taylor series of $\sqrt{I+A}$ w.r.t. $A$ (under the assumption that the root branch is taken so that $\sqrt{I}=I$ ), one can easily notice that the symmetry of matrix $\left(g^{-1} A\right)$ together with the symmetry of $g$ leads to the symmetry of $\left(g^{-1} \sqrt{I+A}\right)$ as all the terms in the series in this case are symmetric. As a result we get $\beta^{\nu \alpha}=\beta^{\alpha \nu}$, so the inverse of it (which we will denote $\hat{\beta}_{\mu \nu}: \hat{\beta}_{\mu \nu} \beta^{\nu \alpha}=\delta_{\nu}^{\alpha}$ ) will also be symmetric: $\hat{\beta}_{\mu \nu}=\hat{\beta}_{\nu \mu}$. Using this fact together with (17), we obtain the equation of motion as the result of variation of (13) w.r.t. $j_{a}^{\mu}$ :

$\partial_{\mu} y^{a}=\hat{\beta}_{\mu \nu} j^{v a}$.

The fact that the metric coincides with induced one (7) follows from it straightforwardly:

$$
\begin{aligned}
\left(\partial_{\mu} y^{a}\right)\left(\partial_{\nu} y_{a}\right) & =\hat{\beta}_{\mu \alpha} j^{\alpha a} \hat{\beta}_{\nu \beta} j^{\beta}{ }_{a} \\
& =\hat{\beta}_{\mu}^{\alpha}\left(\delta_{\alpha}^{\beta}+A_{\alpha}{ }^{\beta}\right) \hat{\beta}_{\beta}^{\gamma} g_{\gamma \nu}=g_{\mu \nu} .
\end{aligned}
$$

Now let us find the expression for EMT of the embedding matter by variation of (13) w.r.t. the metric $g_{\mu \nu}$. Noticing that satisfaction of the EoM (18) implies that

$$
\begin{aligned}
j_{a}^{\mu} \partial_{\mu} y^{a} & =j_{a}^{\mu} \hat{\beta}_{\mu \nu} j^{\nu a}=\hat{\beta}_{\mu}{ }^{\nu}\left(\delta_{v}^{\mu}+{A_{\nu}}^{\mu}\right) \\
& =\operatorname{tr}\left(\beta^{-1}(I+A)\right)=\operatorname{tr} \beta
\end{aligned}
$$

and using (17), we obtain

$\tau^{\mu \nu}=\hat{\beta}_{\alpha}{ }^{\mu} j_{a}^{\nu} j^{\alpha a}=\left(g^{\nu \alpha}+A^{\nu \alpha}\right) \hat{\beta}_{\alpha}^{\mu}=\beta^{\mu \nu}$.

Taking into account the relation $\tau^{\mu \nu} \partial_{v} y^{a}=j^{\mu a}$ according to (18) one can see that Eq. (14) turns out to coincide with (10). As a result we conclude that the embedding gravity given by 
the action (11), (13), completely reproduces the equations of motion of the embedding theory (7), (9), and (10).

The physical meaning of the independent variables $j_{a}^{\mu}$ and $y^{a}$ in the action of embedding matter (13) can be understood more easily than in the action (12): $j_{a}^{\mu}$ at given $a$ can be treated as a conserving (according to (14)) current density of a certain type of matter, whereas the $y^{a}$ turn out to be Lagrange multipliers providing this conservation. Such a form of the action with the Lagrange multiplier, providing current density conservation

$S=\int d^{4} x \sqrt{-g}\left(j^{\mu} \partial_{\mu} \lambda-\sqrt{j^{\mu} j^{\nu} g_{\mu \nu}}\right)$,

can be used in the description of a perfect fluid with no vorticity [3]. The similarity between the action of the embedding matter (13) and the potentially moving perfect fluid (22) is not confined to the presence of Lagrange multipliers. Indeed, their structure is completely identical, including the presence of the root. The only difference is the presence of an additional index of the current density in (13), so (13) simply coincides with (22) if $N=1$, since $\operatorname{tr} \sqrt{j_{\mu} j^{\alpha}}=\sqrt{j_{\mu} j^{\mu}}$ if the vector $j^{\mu}$ is timelike. Note that the theory which appears when one uses (22) as $S^{\text {add }}$ in the action (11) turns out to be equivalent to mimetic gravity.

The necessity of the consideration of the continuity equation $D_{\mu} j^{\mu}=0$ through addition of the term with Lagrange multiplier in the action of perfect fluid is a consequence of the continuous limit. If, on the contrary, one considers the matter as a set of individual particles which have definite worldlines, the continuity is present automatically. If one could construct an analogous "microscopic" description of the embedding matter which automatically provides the continuity equation (14), then it would be unnecessary to add the Lagrange term with $y^{a}$ to the action (13). Unfortunately, it is still unclear how to do so. The only thing that can be noted immediately is the possibility to completely exclude $y^{a}$ (which plays the role of the Lagrange multiplier in this case) from the equations of motion by rewriting (18) in the equivalent form

$D_{\alpha}\left(\hat{\beta}_{\beta \mu} j_{a}^{\mu}\right)-D_{\beta}\left(\hat{\beta}_{\alpha \mu} j_{a}^{\mu}\right)=0$

(the covariant derivatives here can be replaced by ordinary ones due to the symmetry of the connection). As a result the embedding function $y^{a}$ turns out to be completely excluded from the equations of motion of the embedding matter. Such a matter then can be described by the conserved (in accordance with (14)) currents $j_{a}^{\mu}$, which also satisfy the equation of motion (23) and contribute (see (21) and (9)) to the Einstein equations:

$G^{\mu \nu}=\varkappa\left(T^{\mu \nu}+\beta^{\mu \nu}\right)$

(we recall that $\beta^{\mu v}$ and $\hat{\beta}_{\mu \nu}$ are mutually inverse and can be expressed through $j_{a}^{\mu}$ and metric).
An interesting observation is that the structure appearing in the action (13) of the embedding matter, namely the square root of the matrix product, is well known by the socalled bimetric gravity theories [29]. In these theories the role of the matrices is played by two independent metrics, whereas in the action (13) one matrix is a metric $g_{\mu \nu}$ and the other is a symmetric quadratic expression $j_{a}^{v} j^{\alpha a}$. Among the different topics of study in bimetric theories the question of using non-standard root branches is considered along with other mathematical aspects connected with the presence of such a singular expression in the theory; see, e.g. [30,31]. Such subtleties in the approach of embedding gravity require additional study.

By analogy with the possibility to write the perfect fluid action (22) in many equivalent ways [3], there are several ways to write an action equivalent (possibly up to certain special solutions) to (13) without the use of matrix root. In this case, however, the number of independent variables increases. It reaches its maximum in the polynomial action

$$
\begin{aligned}
S_{3}^{\text {add }}= & \int d^{4} x \sqrt{-g} \\
& \times\left(j_{a}^{\mu} \partial_{\mu} y^{a}-\beta^{\mu v} g_{\mu \nu}+\frac{1}{2} \lambda_{\alpha \beta}\left(\beta^{\alpha \gamma} g_{\gamma \delta} \beta^{\delta \beta}-j_{a}^{\alpha} j^{\beta a}\right)\right) .
\end{aligned}
$$

Here the independent variables are $j_{a}^{\mu}, y^{a}$ and the symmetric tensors $\beta^{\mu \nu}, \lambda_{\alpha \beta}$. Variation w.r.t. them gives (besides the continuity equation (14))

$$
\begin{aligned}
& \lambda_{\mu \alpha} \beta^{\alpha \beta} g_{\beta \nu}+g_{\mu \alpha} \beta^{\alpha \beta} \lambda_{\beta \nu}=2 g_{\mu \nu}, \\
& \beta^{\alpha \gamma} g_{\gamma \delta} \beta^{\delta \beta}=j_{a}^{\alpha} j^{\beta a}, \quad \partial_{\mu} y^{a}=\lambda_{\mu \nu} j^{v a} .
\end{aligned}
$$

The first of them has the solution $\lambda_{\mu \nu}=\beta_{\mu \nu}^{-1}$, whose satisfaction leads to the fact that (26) reproduces (18) and (19). It is also easy to check that in this case variation w.r.t. the metric leads to the Einstein equation (24). It should be noted that at the certain special values of $j_{a}^{\mu}$ the solution of the first equation in (26) is not unique, which leads to the special solutions which are absent in the standard formulation of the embedding theory. Such solutions possibly correspond to some solutions of (13) with a non-standard choice of the root branch.

The polynomial action (25) is no more than quadratic w.r.t. $j_{a}^{\mu}$ and $\beta^{\mu \nu}$; moreover, there are no derivatives of these variables. By excluding certain quantities from (25) one can obtain simpler (i.e. with smaller number of variables), but equivalent forms of the action. If one varies the action (25) w.r.t. $\beta^{\mu \nu}$, obtaining the first equation of (26), then solve this equation for it as $\beta^{\mu \nu}=\lambda^{-1 \mu \nu}$, again omitting the abovementioned special solutions, and substituting the result back in the action, one is led to 


$$
\begin{aligned}
S_{4}^{\mathrm{add}}= & \int d^{4} x \sqrt{-g} \\
& \times\left(j_{a}^{\mu} \partial_{\mu} y^{a}-\frac{1}{2} \lambda^{-1 \mu \nu} g_{\mu \nu}-\frac{1}{2} \lambda_{\mu \nu} j_{a}^{\mu} j^{v a}\right) .
\end{aligned}
$$

One can show that further exclusion of $\lambda_{\mu \nu}$ from (27) leads to (13). If one instead excludes $j_{a}^{\mu}$ from (27), then $j_{a}^{\mu}=\lambda^{-1 \mu \nu} \partial_{\nu} y_{a}$ and (27) transforms to the above action (12) where $\tau^{\mu \nu}$ is replaced by $\lambda^{-1 \mu \nu}$.

Alternatively, the variable $j_{a}^{\mu}$ can be excluded from (25) in the first place. It gives the latter equation in (26) and leads to another form of the action:

$$
\begin{aligned}
S_{5}^{\text {add }}= & \int d^{4} x \sqrt{-g}\left(\frac{1}{2} \lambda^{-1 \mu \nu}\left(\partial_{\mu} y^{a}\right)\left(\partial_{\nu} y_{a}\right)\right. \\
& \left.+\frac{1}{2} \lambda_{\alpha \beta} \beta^{\alpha \gamma} g_{\gamma \delta} \beta^{\delta \beta}-\beta^{\mu \nu} g_{\mu \nu}\right) .
\end{aligned}
$$

Further exclusion of $\lambda^{\mu \nu}$ from this action leads to the form of the action which again contains a matrix root:

$$
\begin{aligned}
S_{6}^{\mathrm{add}}= & \int d^{4} x \sqrt{-g} \\
& \times\left(\operatorname{tr} \sqrt{\left(\partial_{\sigma} y^{a}\right)\left(\partial_{\alpha} y_{a}\right) \beta^{\alpha \gamma} g_{\gamma \delta} \beta^{\delta \beta}}-\beta^{\mu v} g_{\mu \nu}\right) .
\end{aligned}
$$

\section{Conclusion and possible development}

Starting from the geometric description of the formulation of gravity proposed by Regge and Teitelboim, the embedding theory, one can rewrite the theory in the form of embedding gravity (11), (13) which is GR with some additional matter. Such a transition is analogous to mimetic gravity (2), (1), when a theory, which was initially formulated as a result of certain change of variables in GR, is rewritten as GR with additional matter resembling a perfect fluid with no vorticity (5), (6). Both approaches can be used in attempts to solve the dark matter and dark energy problems: for the mimetic gravity approach, see [10]; for the embedding theory approach this question was studied in the FRW approximation in Refs. [32-34].

The approach of the embedding theory has a clear geometrical meaning: spacetime is treated as a surface in the flat bulk, whereas the mimetic gravity is based on the change of variables (1), which is constructed artificially to separate the conformal mode of the metric. In the original formulation of mimetic gravity the appearing fictional matter has very simple properties (it is a perfect fluid with potential motion), so the model must be modified (e.g. by introduction of additional parameters; see the Introduction) to explain dark matter. On the contrary, the matter appearing in the embedding gravity approach is highly nontrivial by itself, though it has some properties in common with a perfect fluid. Therefore the attempt to explain dark matter (and possibly dark energy) through embedding gravity with the above action seems promising. An especially interesting problem is the construction of the above-mentioned (see before (23)) "microscopic" description of the embedding matter, which could automatically provide satisfaction of continuity equation (14) for a matter current.

One can use the mentioned similarity between perfect fluid and embedding matter to understand its properties better. If the independent current density $j_{a}^{\mu}$ is nonzero only at a single value of the index $a$ (e.g. $j_{a}^{\mu}=j^{\mu} \delta_{a}^{0}$ ), then the embedding matter precisely becomes a perfect fluid; see after (22). Therefore one can analyze the equations of embedding gravity in the framework of a "non-relativistic" approximation from the point of view of the ambient space (for each value of the $\mu$ ambient space vector $j_{a}^{\mu}$ its 0 th component prevails):

$j_{a}^{\mu}=j^{\mu} \delta_{a}^{0}+h_{a}^{\mu}$,

where $h_{a}^{\mu}$ is a small perturbation. In zeroth order of $h_{a}^{\mu}$, Eq. (23) (containing the inverse quantity $\hat{\beta}_{\mu \nu}$, which needs to be regularized) reduces to the geodesic equation for a normalized velocity vector of the main component of the embedding matter, $u_{\mu}=\hat{\beta}_{\mu \nu} j^{\mu}$; see [3]. However, the perturbative analysis turns out to be a nontrivial problem because of nonsmooth dependence of $\beta_{\mu}^{\alpha}$ and $\hat{\beta}_{\mu \nu}$ on $j_{a}^{\mu}$ in the expansion (30) (we recall that $\beta_{\mu}{ }^{\alpha}$ is a result of the matrix root taken in (13) and $\hat{\beta}_{\mu \nu}$ being the inverse). Such an analysis requires additional study and lies beyond the scope of the present paper.

This nontriviality is largely related to the non-linearizability of the Regge-Teitelboim equation (8) (their properties were discussed, e.g., in [13]) in the original formulation of the embedding theory when the most natural background embedding function $y^{a}(x)$ is chosen which corresponds to a 4D plane. Also note that because of this non-linearizability it is difficult to use the results obtained for the dynamics of the embedding matter in the framework of the FRW symmetry [32-34] as a basis on the perturbation theory aimed to transcend this symmetry.

As a final remark we note that if one considers the action

$\tilde{S}=S^{\mathrm{b}}+S^{\mathrm{add}}, \quad S^{\mathrm{b}}=-T \int d^{4} x \sqrt{-g}$

instead of (11) ( $T$ is the brane tension) with any kind of $S^{\text {add }}$ discussed in Sect. 2, one obtains the description of a 3-brane; and lowering the dimension from 4 to 2 leads to the NambuGoto bosonic string. To prove this, one needs to write the equation of motion of the brane described by the embedding function $y^{a}(x)$ with the action $S^{\mathrm{b}}\left[g_{\mu \nu}\right]$, where the metric is given by (7), in the form analogous to (10):

$D_{\mu}\left(g^{\mu \nu} \partial_{\nu} y^{a}\right)=0$. 
On the other side one must take into account that for all forms of $S^{\text {add }}$ considered in Sect. 2 the equations of motion provide the existence of (7) for the metric and lead to (10), and the variation of $\tilde{S}$ w.r.t. metric gives the equations $T g^{\mu \nu}+\tau^{\mu \nu}=$ 0 instead of the Einstein ones.

If (12) is chosen as $S^{\text {add }}$ and the dimension is set to 2, then the bosonic string action turns out to have the form

$\tilde{S}=-T \int d^{2} x\left(\sqrt{-g}+\frac{1}{2}\left(\left(\partial_{\mu} y^{a}\right)\left(\partial_{\nu} y_{a}\right)-g_{\mu \nu}\right) \tilde{\tau}^{\mu \nu}\right)$,

where the new independent variable $\tilde{\tau}^{\mu \nu}=-\sqrt{-g} \tau^{\mu \nu} / T$ is introduced. Interestingly enough, it is this action that leads to the Polyakov action [35] in a most simple way-through exclusion of the variable $g_{\mu \nu}$. Indeed, the variation w.r.t. $g_{\mu \nu}$ gives the equation $\sqrt{-g} g^{\mu \nu}=\tilde{\tau}^{\mu \nu}$. In two dimensions it leads to the condition det $\tilde{\tau}=-1$, and usage of the obtained equation in (33) allows one to rewrite the action in the following form:

$\tilde{S}=-\frac{T}{2} \int d^{2} x \tilde{\tau}^{\mu \nu}\left(\partial_{\mu} y^{a}\right)\left(\partial_{\nu} y_{a}\right)$.

Taking into account that an arbitrary $2 \times 2$ matrix satisfying $\operatorname{det} \tilde{\tau}=-1$ can be written as $\tilde{\tau}^{\mu \nu}=\sqrt{-h} h^{\mu \nu}$, where $h_{\mu \nu}$ is an arbitrary auxiliary metric, the action can be presented as

$\tilde{S}=-\frac{T}{2} \int d^{2} x \sqrt{-h} h^{\mu \nu}\left(\partial_{\mu} y^{a}\right)\left(\partial_{\nu} y_{a}\right)$,

i.e. in the Polyakov form. Since a classical theory of the bosonic string is much simpler than GR, a theory with an action $\tilde{S}(31)$ and different forms of $S^{\text {add }}$ from Sect. 2 can be a toy model useful for the analysis of embedding gravity with the same $S^{\text {add }}$ in the action (11).

Acknowledgements The authors are grateful to A. Golovnev and A. Starodubtsev for useful discussions. The work of one of the authors (A. A. Sheykin) was supported by RFBR Grant N 18-31-00169.

Open Access This article is distributed under the terms of the Creative Commons Attribution 4.0 International License (http://creativecomm ons.org/licenses/by/4.0/), which permits unrestricted use, distribution, and reproduction in any medium, provided you give appropriate credit to the original author(s) and the source, provide a link to the Creative Commons license, and indicate if changes were made.

Funded by SCOAP ${ }^{3}$.

\section{References}

1. A.H. Chamseddine, V. Mukhanov, J. High Energy Phys. 11, 135 (2013). arXiv: 1308.5410

2. A. Golovnev, Phys. Lett. B 728, 39-40 (2014). arXiv: 1310.2790

3. S.A. Paston, Phys. Rev. D 96, 084059 (2017). arXiv:1708.03944
4. A.O. Barvinsky, J. Cosmol. Astropart. Phys. 01, 014 (2014). arXiv: 1311.3111

5. K. Hammer, A. Vikman, Many Faces of Mimetic Gravity (2015). arXiv: 1512.09118

6. A.H. Chamseddine, V. Mukhanov, A. Vikman, J. Cosmol. Astropart. Phys. 06, 017 (2014). arXiv:1403.3961

7. E. A. Lim, I. Sawicki, A. Vikman, J. Cosmol. Astropart. Phys. 05, 012 (2010). arXiv:1003.5751

8. L. Mirzagholi, A. Vikman, J. Cosmol. Astropart. Phys. 06, 028 (2015). arXiv:1412.7136

9. Sh Hirano, S. Nishi, T. Kobayashi, J. Cosmol. Astropart. Phys. 07, 009 (2017). arXiv: 1704.06031

10. L. Sebastiani, S. Vagnozzi, R. Myrzakulov, Adv. High Energy Phys. 2017, 3156915 (2017). arXiv:1612.08661

11. J.D. Bekenstein, Phys. Rev. D 48(8), 3641-3647 (1993). arXiv:gr-qc/9211017

12. N. Deruelle, J. Rua, J. Cosmol. Astropart. Phys. 09, 1409 (2014). arXiv: 1407.0825

13. A.A. Sheykin, S.A. Paston, AIP Conf. Proc. 1606, 400 (2014). arXiv: 1402.1121

14. T. Regge, C. Teitelboim, General relativity à la string: a progress report. In Proceedings of the First Marcel Grossmann Meeting, Trieste, Italy, 1975, edited by R. Ruffini, 77-88 (North Holland, Amsterdam, 1977). arXiv:1612.05256

15. A. Friedman, J. Math. Mech. 10, 625 (1961)

16. S. Deser, F.A.E. Pirani, D.C. Robinson, Phys. Rev. D 14(12), 33013303 (1976)

17. M. Pavsic, Phys. Lett. A 107, 66-70 (1985)

18. V. Tapia, Class. Quant. Gravit. 6, L49 (1989)

19. M.D. Maia, Class. Quant. Gravit. 6, 173-183 (1989)

20. F.B. Estabrook, R.S. Robinson, H.R. Wahlquist, Class. Quant. Gravit. 16, 911-918 (1999)

21. D. Karasik, A. Davidson, Phys. Rev. D 67, 064012 (2003). arXiv:gr-qc/0207061

22. S.A. Paston, V.A. Franke, Theor. Math. Phys. 153(2), 1582-1596 (2007). arXiv:0711.0576

23. R. Cordero, A. Molgado, E. Rojas, Phys. Rev. D 79, 024024 (2009). arXiv:0901.1938

24. L.D. Faddeev, Theor. Math. Phys. 166, 3, 279-290 (2011). arXiv:0906.4639. arXiv:0911.0282. arXiv:1003.2311

25. S.A. Paston, Theor. Math. Phys. 169(2), 1611-1619 (2011). arXiv: 1111.1104

26. S.A. Paston, E.N. Semenova, V.A. Franke, A.A. Sheykin, Gravit. Cosmol. 23, 1, 1-7 (2017). arXiv:1705.07361

27. M. Pavsic, V. Tapia, Resource letter on geometrical results for Embeddings and Branes (2000). arXiv:gr-qc/0010045

28. M. Pavsic, Class. Quant. Gravit. 2, 869 (1985). arXiv:1403.6316

29. A. Schmidt-May, M. von Strauss, J. Phys. A: Math. Theor. 49, 18 183001 (2016). arXiv:1512.00021

30. A. Golovnev, F. Smirnov, J. High Energy Phys. 6, 130 (2017). arXiv: 1704.08874

31. S.F. Hassan, M. Kocic, J. High Energy Phys. 5, 99 (2018). arXiv: 1706.07806

32. A. Davidson, Class. Quant. Gravit. 16, 653 (1999). arXiv:gr-qc/9710005

33. A. Davidson, D. Karasik, Y. Lederer, Cold Dark Matter from Dark Energy (2001). arXiv:gr-qc/0111107

34. S.A. Paston, A.A. Sheykin, Int. J. Mod. Phys. D 21(5), 1250043 (2012). arXiv:1106.5212

35. A.M. Polyakov, Phys. Lett. B 103(3), 207-210 (1981) 